\title{
Quadratic magnetic field dependence of magnetoelectric photocurrent
}

\author{
Junfeng Dai (戴俊峰), ${ }^{1}$ Hai-Zhou Lu (卢海舟), ${ }^{1,2}$ Shun-Qing Shen (沈顺清), ${ }^{1,2}$ Fu-Chun Zhang (张富春), ${ }^{1,2}$ and \\ Xiaodong Cui (崔晓冬) $)^{1, *}$ \\ ${ }^{1}$ Department of Physics, The University of Hong Kong, Pokfulam Road, Hong Kong, China \\ ${ }^{2}$ Centre of Theoretical and Computational Physics, The University of Hong Kong, Pokfulam Road, Hong Kong, China
}

(Received 19 December 2010; published 8 April 2011)

\begin{abstract}
We experimentally study the spin and electric photocurrents excited by a linearly polarized light via direct interband transitions in an InGaAs/InAlAs quantum well. In the absence of a magnetic field, the linearly polarized light induces a pure spin current due to the spin-orbit coupling, which may be transformed into a measurable electric current by applying an in-plane magnetic field. The induced electric photocurrent is linear with the in-plane magnetic field. Here, we report a quadratic magnetic field dependence of the photocurrent in the presence of an additional perpendicular component of the magnetic field. We attribute the observation to the Hall effect of magnetoelectric photocurrent.
\end{abstract}

DOI: 10.1103/PhysRevB.83.155307

PACS number(s): 72.25.Dc, 73.63.Hs, 78.67.De

Generation and manipulation of spin currents are of fundamental interest in the field of spintronics. The optical injection of pure spin current can be realized by optical excitation in semiconductors with strong spin-orbit coupling, which manipulates electron spin via optical selection rules and generates a spin related current through a spin-momentum interaction. ${ }^{1-10}$ One particular example is the linearly polarized optical excitation in two-dimensional III-V semiconductors. ${ }^{9,11,12}$ For a two-dimensional electron gas (2DEG) system, the spin-orbit coupling lifts the spin degeneracy in $k$ space and generates a linear spin-momentum correlated term. A linearly polarized light combined with the spin-split band structure generates the same amount of carriers with opposite spins and velocities, leading to a spin photocurrent, ${ }^{13}$ accompanied by no electric current if neither in-plane inhomogeneity nor the photon drag exists. The measurement of the spin photocurrent may be realized by means of applying an external magnetic field. An in-plane magnetic field induces an imbalance of photoexcited carriers with opposite spins in the spin-split bands, resulting in an electric photocurrent. This magnetic induced electric current shows a linear dependence on the magnetic field at low field by independent groups, although symmetry analysis cannot exclude a nonlinear dependence. In this paper, we report a quadratic magnetic field dependence of magnetoelectric photocurrent in an InGaAs/InAlAs 2DEG strip, in the presence of an additional perpendicular component of magnetic field. We attribute the observation to the Hall effect of magnetoelectric photocurrent.

The experiments were performed on a $40 \mathrm{~nm}$ thick $\mathrm{In}_{x} \mathrm{Ga}_{1-x} \mathrm{As} / \mathrm{InAlAs}$ quantum well (QW) grown on semiinsulating [001] InP substrate by molecular beam epitaxy. The mobility and carrier density were determined as $\mu_{m}=12000$ $\mathrm{cm}^{2} \mathrm{~V}^{-1} \mathrm{~s}^{-1}$ and $n_{e}=2.2 \times 10^{12} \mathrm{~cm}^{-2}$ at room temperatures by low field Hall measurements. The InGaAs QW is modulated doped on the top side only, with graded indium composition from $x=0.53$ to 0.59 . Both the structure inversion asymmetry (SIA) and bulk inversion asymmetry (BIA) present in the quantum well. The graded indium doping further enhances the strong SIA, which is the dominant spin-orbit coupling. In the measurements, $x$ and $y$ axes are defined along the [11 0$]$ and [110] crystallographic directions, respectively. In this coordinate system, the spin-orbit coupling in the presence of both SIA and BIA is described by an anisotropic Rashba model. ${ }^{14-16}$ On average, the Rashba coefficient is about $\alpha=4.3 \times 10^{-12} \mathrm{eV} \cdot \mathrm{m}$ determined from the beating pattern of Shubnikov-de Hass oscillation at $2.5 \mathrm{~K}$. The band gap of 2DEG extracted from the photomodulated differential reflectivity spectrum is about $0.764 \mathrm{eV}$ at $77 \mathrm{~K}$. The $180 \mu \mathrm{m}$ wide and $1800 \mu \mathrm{m}$ long 2DEG strip along the [1110] direction was prepared by standard photolithography and wet etching. Electric access is formed at the strip ends through Ohmic contact with annealed $\mathrm{Ni} / \mathrm{Ge} / \mathrm{Au} / \mathrm{Ge} / \mathrm{Ni} / \mathrm{Au}$ alloy.

The schematic of the experimental setup is shown in Fig. 2(a). As suggested in the photomodulated reflectivity spectrum, a linearly polarized light of $1480 \mathrm{~nm}(0.838 \mathrm{eV})$ from a DFB packed laser diode is employed as an exciting source for the direct interband transition. The pump light passes through a chopper with a frequency of $2 \mathrm{kHz}$, a polarizer and a half wave-plate which is used to rotate the light polarization, and then is focused through a $10 \times$ objective normally onto the sample mounted inside a cryostat. The diameter of the focused beam spot is around $30 \mu \mathrm{m}$ and the beam completely shines on the central part of the 2DEG channel in order to avoid any edge effects. The electric photocurrent flowing along the channel is collected via voltage drop on a loaded resistor with a lock-in amplifier. All photocurrent measurements are carried out in vacuum condition $\left(\sim 10^{-4} \mathrm{~Pa}\right)$ at liquid nitrogen temperature.

When a linearly polarized light is normally incident on the sample in the absence of magnetic field, no measurable current along the channel is observed above the background noise of tens of picoamperes. This is expected for a zinc-blended QW grown along the [001] direction under a linearly polarized excitation at normal incidence where circular ${ }^{17}$ or linear photogalvanic effect ${ }^{18}$ or photon-drag effect ${ }^{19}$ is safely excluded. While an in-plane magnetic field is applied, an electric photocurrent $J_{x}$ is generated and is linearly proportional to the magnetic field. The electric current also shows strong dependence on the light polarization angle. The phenomenon can be summarized by

$$
J_{x}\left(B_{x}, B_{y}, \theta\right)=c_{0} B_{y}+c_{y} B_{y} \cos 2 \theta+c_{x} B_{x} \sin 2 \theta,
$$

where $c_{0}=6.00 \times 10^{-8} \mathrm{~A} /(\mathrm{T} \cdot \mathrm{W}), c_{x}=0.40 \times 10^{-8} \mathrm{~A} /(\mathrm{T} \cdot \mathrm{W})$, and $c_{y}=-0.73 \times 10^{-8} \mathrm{~A} /(\mathrm{T} \cdot \mathrm{W})$ are constants, $\theta$ is the angle 

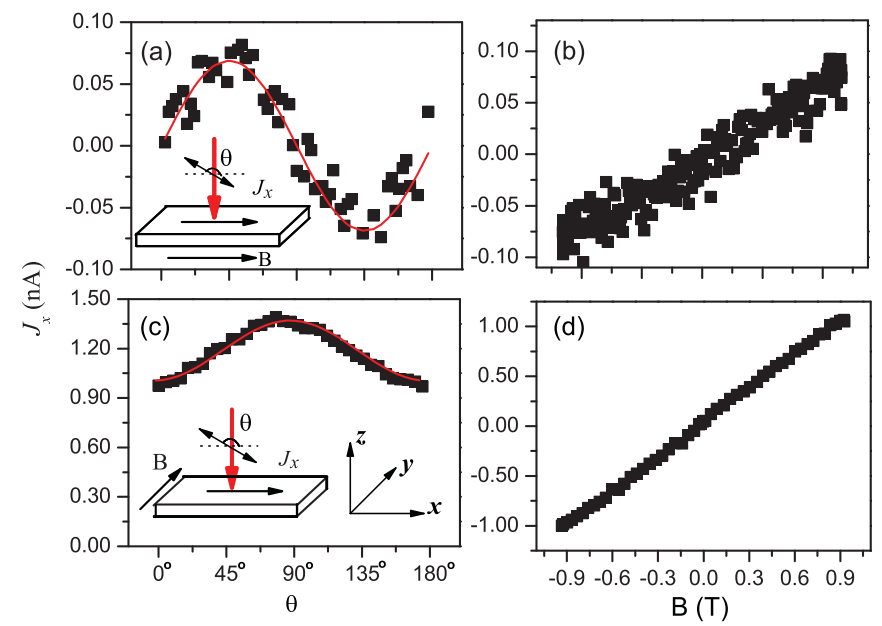

FIG. 1. (Color online) Electric photocurrent $J_{x}$ as a function of the polarization angle $\theta$ and in-plane magnetic field $B$, along $x$ axis [(a)-(b)], and along $y$ axis [(c)-(d)]. $B=0.9 \mathrm{~T}$ in (a) and (c). $\theta=45^{\circ}$ in (b), and $\theta=0^{\circ}$ in (d). The red curves represent fittings assuming $\sin 2 \theta$ (a) and $\cos 2 \theta$ (c) dependence.

of the light polarization with respect to the 2DEG strip, and $B_{x}$ and $B_{y}$ stand for the magnetic field along the $x$ axis ([1 $\left.\left.\overline{1} 0\right]\right)$ and $y$ axis([110]), respectively. Note that $c_{0}$ is significantly larger than $c_{x}$ and $c_{y}$. The difference in magnitudes of $c_{x}$ and $c_{y}$ implicitly reflects the anisotropic strength of spin-orbit coupling along specific crystallographic directions. ${ }^{14-16}$ The angle of light polarization and magnetic field dependence of electric photocurrent are shown in Fig. 1.

The observed linear dependence of the photocurrents on the in-plane magnetic fields is consistent with all the previous experiments in similar structures. ${ }^{2,11,12}$ They originate from the unbalanced spin photocurrents induced by the Zeeman energy of the in-plane magnetic field. For a zinc-blende structure with strong SIA along the [001] direction (which is cataloged into the $C_{2 v}$ group), the spin-orbit coupling lifts the spin degeneracy of the conduction bands, and splits them into + and - bands. Because the spin-orbit coupling respects time reversal symmetry, for any specific direction, the linear light excites two degenerated pairs of carriers. Each pair of carriers processes opposite velocities and opposite spin orientations. As a result, linearly polarized light will not induce electric currents, but finite pure spin photocurrents. Upon applying an in-plane magnetic field, the Zeeman splitting will break the balance of the spins as well as the velocities of photocarriers and consequently an electric current emerges. Moreover, the dependence of the photocurrents on the polarization angle originates from the anisotropy of the photoexcited carrier density, which is the consequence of the quantum interference between the photoexcited carriers excited by the two circular components of the linearly polarized light. A microscopic model is described in Ref. 12.

To examine the effect of coexisting in-plane and outof-plane magnetic fields, we tilt the magnetic field in the $x-z$ plane (azimuth angle $\Phi=0$ ) by polar angle $\Theta$, $\vec{B}=(B \sin \Theta, 0, B \cos \Theta)$. Figures 2(b) and 2(c) present the magnetoelectric photocurrent as functions of the magnetic field $\mathrm{B}$ where the light polarization $\theta$ is fixed along the
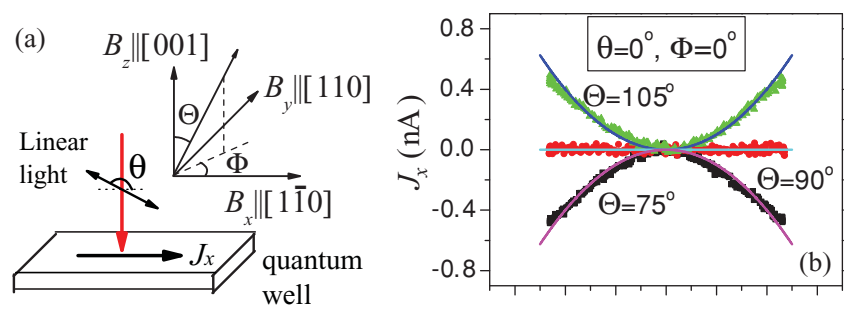

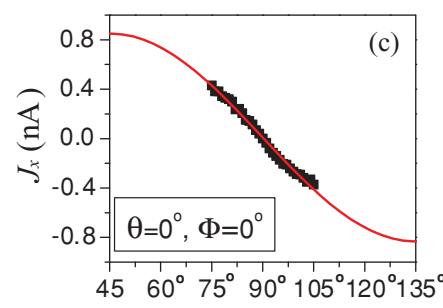

$\Theta$

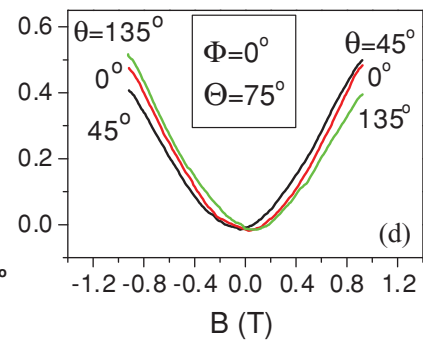

$\mathrm{B}(\mathrm{T})$
FIG. 2. (Color online) (a) Schematic illustration of the setup, in which a linearly polarized light (downward arrow) is normally incident into the 2DEG strip lying in $x-y$ plane. $\theta$ denotes the angle between light polarization and $x$ axis. The $x, y$, and $z$ axes are defined along [1 10], [110], and [001] crystallographic directions, respectively. (b) Magnetoelectric photocurrents $J_{x}$ as a function of magnetic field $B$ at magnetic field tilt angles $\Theta=75^{\circ}, 90^{\circ}$, and $105^{\circ}$ where the light polarization is fixed along $x$ axis ([1ํㅣㅇ direction). (c) $J_{x}$ as a function of magnetic field tilt angles $\Theta$ (dot) at $|B|=0.8 \mathrm{~T}$ and a fit curve assuming a $\sin 2 \Theta$ dependence. (d) $J_{x}$ as a function of magnetic field $B$ under a fixed magnetic tilt angle $\Theta=75^{\circ}$ at various light polarizations.

$x$ axis, which clearly shows a quadratic dependence on the magnetic field. For a fixed polar angle $\Theta$, the change of light polarization $\theta$ only translates the quadric curve without measurable distortion, as shown in Fig. 2(d). We could brief the magnetoelectric photocurrent as a function of magnetic field, polar angle $\Theta$, and light polarization $\theta$ by

$$
J_{x}=c_{x} B_{x} \sin 2 \theta+c_{z x} B_{x} B_{z},
$$

where $c_{x}$ and $c_{z x}$ are constants linearly scaling with the light intensity, $B_{x}=B \sin \Theta$, and $B_{z}=B \cos \Theta$. The first term is contributed by the translation of current extremum with the change of light polarization, and the second represents the quadratic magnetic field dependence.

If the magnetic field is tilted in the $y-z$ plane around the $x$ axis where azimuth angle $\Phi=90^{\circ}$ and $\vec{B}=(0, B \sin \Theta, B \cos \Theta)$, the magnetoelectric photocurrent demonstrates a linear dependence on $B_{y}$, but negligible dependence on $B_{z}$ within the low field range $(\leqslant 1 \mathrm{~T})$. Figure 3 shows that the electric photocurrent dependence on the magnetic field $B$ gradually evolves from linear to quadratic as the azimuth angle of the magnetic field changes from $\Phi=90^{\circ}$ to $0^{\circ}$ with fixed nonzero polar angle $\Theta$, where $\vec{B}=(B \sin \Theta \cos \Phi, B \sin \Theta \sin \Phi, B \cos \Theta)$. Summarizing all the above, we can formulate our observation as follows:

$$
J_{x}=\left(c_{0}+c_{y} \cos 2 \theta\right) B_{y}+c_{x} B_{x} \sin 2 \theta+c_{z x} B_{x} B_{z} .
$$

Phenomenologically, up to the quadratic terms in magnetic fields, the magnetoelectric photocurrent $J_{\alpha}$ induced by secondorder nonlinear optical excitations in a structure belonging to 


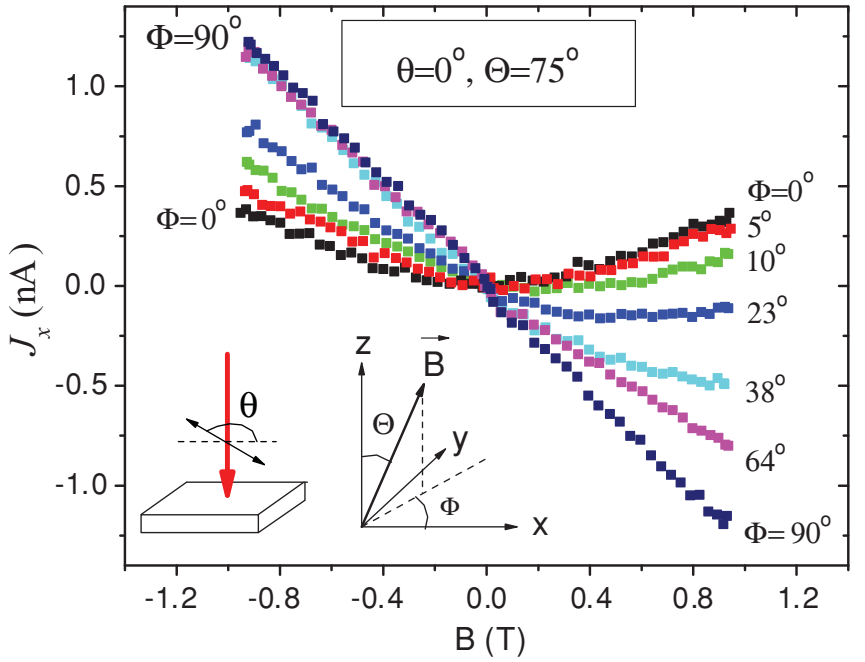

FIG. 3. (Color online) Magnetoelectric photocurrents $J_{x}$ as a function of magnetic field $B$ at different magnetic field tilt angles $\Phi$ with fixed $\Theta=75^{\circ}$, where the light polarization is fixed along $x$ axis ([1]10] direction).

the $C_{2 v}$ group can be generally summarized as (details can be found in the Appendix)

$$
\begin{aligned}
J_{x}= & \left(c_{0}+c_{y} \cos 2 \theta\right) B_{y}+c_{x} B_{x} \sin 2 \theta \\
& +\left(c_{z x}^{0}+c_{z x}^{\cos } \cos 2 \theta\right) B_{x} B_{z}+c_{z y}^{\sin } B_{y} B_{z} \sin 2 \theta,
\end{aligned}
$$

where $\cos 2 \theta$ and the $\theta$-independent terms stem from $E_{x}^{2}$ and $E_{y}^{2}$, and $\sin 2 \theta$ from $E_{x} E_{y}$, the second-harmonic terms of the polarization electric fields of the laser.

Now we explain the microscopic picture of the $B^{2}$ term in the current formula. From both symmetry analysis and the microscopic model, ${ }^{12}$ we already know that magnetoelectric current along the $x$ direction can be induced by either longitudinal or perpendicular in-plane magnetic fields. Based on the same theories, there should be a current flowing along the $y$ direction in the presence of only in-plane magnetic fields $B_{x}$ and $B_{y}$, as shown in Fig. 4. This current can be described by

$$
J_{y}=\left(c_{y x}^{0}+c_{y x}^{\cos } \cos 2 \theta\right) B_{x}+c_{y y}^{\sin } B_{y} \sin 2 \theta,
$$

where $c_{y x}^{0}, c_{y x}^{\cos }$, and $c_{y y}^{\text {sin }}$ are phenomenological coefficients. Since the experimental setup only measures the current along the $x$ direction, this transverse current cannot be detected, unless a magnetic field $B_{z}$ is applied along the $z$ direction. $B_{z}$ will draw $J_{y}$ to the $x$ direction due to the Hall effect, leading to the $B^{2}$ contributions to $J_{x}$, i.e.,

$$
\begin{aligned}
J_{y} & =\left(c_{y x}^{0}+c_{y x}^{\cos } \cos 2 \theta\right) B_{x}+c_{y y}^{\sin } B_{y} \sin 2 \theta \\
& \Rightarrow J_{x}=\left(c_{z x}^{0}+c_{z x}^{\cos } \cos 2 \theta\right) B_{x} B_{z}+c_{z y}^{\sin } B_{y} B_{z} \sin 2 \theta .
\end{aligned}
$$

This picture is schematically illustrated in Fig. 4. As described in Eq. (6), the second and third terms, $c_{y x}^{\cos } B_{x} \cos 2 \theta$ and $c_{y y}^{\sin } B_{y} \sin 2 \theta$, should also lead to a $B^{2}$ contribution in the presence of $B_{z}$ through the Hall effect. However, as shown in Fig. 3, no quadratic magnetic field dependence is observed at magnetic field tilt angle $\Phi=90^{\circ}$ and $\Theta=75^{\circ}$, and in Fig. 2, no $\cos 2 \theta$ dependence is detected in the $B_{x} B_{z}$ term. There are two main reasons. First, the polarization-independent

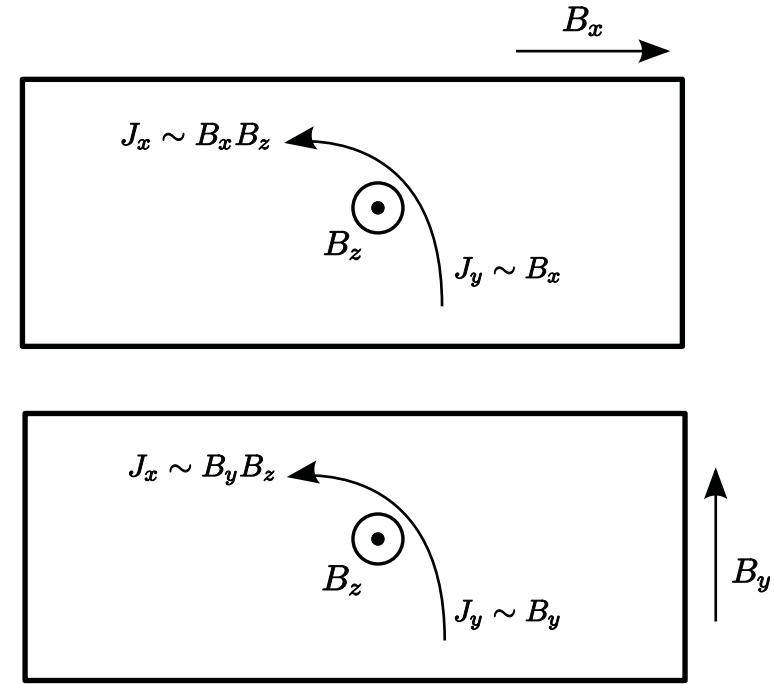

FIG. 4. The origin of the quadratic magnetic field dependence of the magnetoelectric photocurrents.

component $c_{y x}^{0}$ of the photocurrent in Eq. (6) is one order of magnitude larger than other two terms, so the photocurrent transformed from $c_{y x}^{\cos } B_{x} \cos 2 \theta$ and $c_{y y}^{\sin } B_{y} \sin 2 \theta$ under the Hall effect is much smaller than that from the first term. Second, the Hall effect at low magnetic fields cannot divert all the photocurrent along the $y$ axis to the $x$ direction. As a result, $c_{z x}^{\cos } B_{x} B_{z} \cos 2 \theta$ and $c_{z y}^{\sin } B_{y} B_{z} \sin 2 \theta$ cannot be detected under our measurement resolution. Employing the Hall effect of magnetoelectric photocurrent, the carrier's density can be estimated by the following equation, $n_{e}=J_{y} B_{z} L / w e V_{x}=$ $2.18 \times 10^{12} \mathrm{~cm}^{-2},{ }^{21}$ where $L$ and $w$ are the length and width of the 2DEG strip, $B_{z}$ is the magnetic field along the $z$ direction, $V_{x}$ is the Hall voltage under $B_{z}$, and $J_{y}$ is the magnetoelectric photocurrent along the $y$ direction under $B_{x}$ and can be approximated by $J_{x}$ induced by $B_{y}$. This result is in good agreement with that by the standard Hall measurement.

In conclusion, we observe the quadratic magnetic field dependence of magnetoelectric photocurrent and attribute the phenomena to the combination of the unbalanced photoexcited carriers with opposite spins induced by the Zeeman energy of the in-plane magnetic field and the Hall effect under out-of-plane magnetic field. The quadratic dependence on

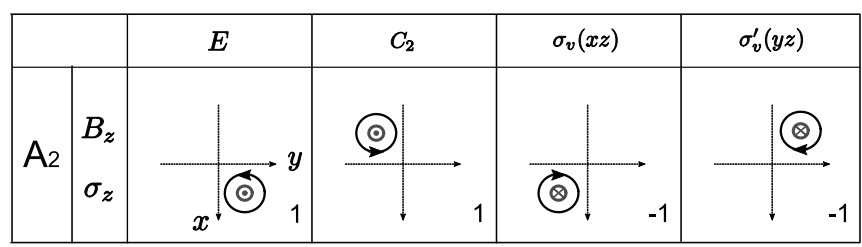

FIG. 5. When axes $x, y$, and $z$ are defined along [110], [110], and [001] crystallographic directions, how the axial vectors $B_{z}$, $\sigma_{z}$ transform under the four symmetry operations of the $C_{2 v}$ point group. $E$ : identical; $C_{2}$ : twofold rotation about $z$ axis; $\sigma_{v}\left(\sigma_{v}^{\prime}\right)$ : mirror reflection with respect to $x z$, $y z$ ) plane. " 1 " or " -1 " on the lower right corner of each panel are the characters of the irreducible representation $\mathrm{A}_{2}$. They also indicate whether the vectors change sign under the symmetry operations. ${ }^{20}$ 
magnetic field may inspire some potential applications in spintronics research.

This work was supported by the Research Grant Council of Hong Kong under Grants No. HKU 10/CRF/08, No. HKU 704107P, and No. HKU701308P.

\section{APPENDIX: DERIVATION OF EQ. (4) FROM SYMMETRY ANALYSIS}

Our quantum well is an inversion-asymmetric zinc blende heterostructure grown along the [001] direction. It has the $C_{2 v}$ point group symmetry, with four symmetry operations and four irreducible representations. ${ }^{20}$ In our measurements, the $x$ and $y$ axes are defined as the [1 10$]$ and [110] crystallographic directions, respectively, and the $x z$ and $y z$ planes coincide with the mirror reflection planes of the $C_{2 v}$ group. As the basis functions, $J_{x}, E_{x}, B_{y}$ transform according to the irreducible representation $\mathrm{B}_{1}, E_{y}, B_{x}$ according to $\mathrm{B}_{2}$, and $B_{z}$ according to $A_{2}$. The physical picture of "vectors transforming according to irreducible representations" has been schematically illustrated in Ref. 16 for the representations $B_{1}$ and $B_{2}$. In Fig. 5, the physical picture for $\mathrm{A}_{2}$ is also supplied.
Phenomenologically, the magnetoelectric photocurrent $J_{\alpha}$ up to the quadratic order of magnetic fields can be generally written as

$$
J_{\alpha}=\chi^{\alpha \beta \gamma \delta} E_{\beta} E_{\gamma} B_{\delta}+\kappa^{\alpha \beta \gamma \delta \eta} E_{\beta} E_{\gamma} B_{\delta} B_{\eta},
$$

where $\alpha, \beta, \gamma, \delta$, and $\eta$ stand for Cartesian coordinates. $B_{\beta}$ and $E_{\gamma}$ are the components of the magnetic field and polarization electric field vector. The nonzero terms of Eq. (A1) require that the vectors on both sides transform in the same manner for all the symmetry operations of the $C_{2 v}$ group. The $\chi$ and $\kappa$ terms in Eq. (A1) will lead to the $B$-linear and $B$-quadratic terms in Eq. (4), respectively. While the $\chi$ term has been illustrated in Ref. 16, here we give examples of the $\kappa$ term. With the help of the product table of the $\mathrm{C}_{2 v}$ group, ${ }^{20}$ we can write arbitrarily

$$
\begin{aligned}
& \mathrm{B}_{1}=\mathrm{B}_{2} \otimes \mathrm{B}_{1} \otimes \mathrm{B}_{1} \otimes \mathrm{A}_{2}, \\
& \mathrm{~B}_{1} \neq \mathrm{B}_{2} \otimes \mathrm{B}_{1} \otimes \mathrm{B}_{2} \otimes \mathrm{A}_{2},
\end{aligned}
$$

which mean that $J_{x}$ can contain terms such as $\kappa^{x y x y z} E_{y} E_{x} B_{y} B_{z}$, but not $\kappa^{x y x x z} E_{y} E_{x} B_{x} B_{z}$. Similarly, by running over $x, y, z$ for each component in Eq. (A1), all the nonzero terms can be found. By using $E_{x} \propto \cos \theta, E_{y} \propto \sin \theta$ and redefining the parameters, we finally obtain Eq. (4). *xdcui@hku.hk

${ }^{1}$ S. D. Ganichev and W. Prettl, J. Phys. Condens. Matter 15, R935 (2003).

${ }^{2}$ S. D. Ganichev, E. L. Ivchenko, V. V. Bełkov, S. A. Tarasenko, M. Sollinger, D. Weiss, W. Wegscheider, and W. Prettl, Nature (London) 417, 153 (2002).

${ }^{3}$ J. Hübner, W. W. Rühle, M. Klude, D. Hommel, R. D. R. Bhat, J. E. Sipe, and H. M. van Driel, Phys. Rev. Lett. 90, 216601 (2003). ${ }^{4}$ M. J. Stevens, A. L. Smirl, R. D. R. Bhat, Ali Najmaie, J. E. Sipe, and H. M. van Driel, Phys. Rev. Lett. 90, 136603 (2003).

${ }^{5}$ H. Zhao, X. Pan, A. L. Smirl, R. D. R. Bhat, Ali Najmaie, J. E. Sipe, and H. M. van Driel, Phys. Rev. B 72, 201302 (2005).

${ }^{6}$ X. D. Cui, S. Q. Shen, J. Li, Y. Ji, W. K. Ge, and F. C. Zhang, Appl. Phys. Lett. 90, 242115 (2007).

${ }^{7}$ B. Zhou and S. Q. Shen, Phys. Rev. B 75, 045339 (2007).

${ }^{8}$ J. Li, X. Dai, S. Q. Shen, and F. C. Zhang, Appl. Phys. Lett. 88, 162105 (2006).

${ }^{9}$ R. D. R. Bhat and J. E. Sipe, Phys. Rev. Lett. 85, 5432 (2000).

${ }^{10}$ V. V. Bełkov, S. D. Ganichev, E. L. Ivchenko, S. A. Tarasenko, W. Weber, S. Giglberger, M. Olteanu, H. P. Tranitz, S. N. Danilov, Petra Schneider, W. Wegscheider, D. Weiss, and W. Prettl, J. Phys. Condens. Matter 17, 3405 (2005).

${ }^{11}$ S. D. Ganichev, V. V. Bełkov, S. A. Tarasenko, S. N. Danilov, S. Giglberger, C. Hoffmann, E. L. Ivchenko, D. Weiss, W. Wegscheider, C. Gerl, D. Schuh, J. Stahl, J. D. Boeck, G. Borghs, and W. Prettl, Nature Phys. 2, 609 (2006).
${ }^{12}$ J. F. Dai, H. Z. Lu, C. L. Yang, S. Q. Shen, F. C. Zhang, and X. D. Cui, Phys. Rev. Lett. 104, 246601 (2010).

${ }^{13} \mathrm{~S}$. Tarasenko and E. Ivchenko, JETP Lett. 81, 231 (2005).

${ }^{14}$ S. D. Ganichev, V. V. Bełkov, L. E. Golub, E. L. Ivchenko, P. Schneider, S. Giglberger, J. Eroms, J. De Boeck, G. Borghs, W. Wegscheider, D. Weiss, and W. Prettl, Phys. Rev. Lett. 92, 256601 (2004).

${ }^{15}$ S. Giglberger, L. E. Golub, V. V. Bełkov, S. N. Danilov, D. Schuh, C. Gerl, F. Rohlfing, J. Stahl, W. Wegscheider, D. Weiss, W. Prettl, and S. D. Ganichev, Phys. Rev. B 75, 035327 (2007).

${ }^{16}$ H. Z. Lu, B. Zhou, F. C. Zhang, and S. Q. Shen, Phys. Rev. B 83, 125320 (2011)

${ }^{17}$ S. D. Ganichev, E. L. Ivchenko, S. N. Danilov, J. Eroms, W. Wegscheider, D. Weiss, and W. Prettl, Phys. Rev. Lett. 86, 4358 (2001).

${ }^{18}$ S. D. Ganichev, S. N. Danilov, V. V. Bełkov, E. L. Ivchenko, M. Bichler, W. Wegscheider, D. Weiss, and W. Prettl, Phys. Rev. Lett. 88, 057401 (2002).

${ }^{19}$ A. D. Wieck, H. Sigg, and K. Ploog, Phys. Rev. Lett. 64, 463 (1990).

${ }^{20}$ M. S. Dresselhaus, G. Dresselhaus, and A. Jorio, Group Theory Application to the Physics of Condensed Matter (Springer-Verlag, Berlin, 2008).

${ }^{21}$ K. Seeger, Semiconductor Physics: An Introduction (Springer, Berlin/London, 1999). 